\title{
Die Quelle zu Rutebeufs Leben der hl. Elisabeth.
}

Es soll der Zweck dieser Arbeit sein, nachzuweisen, dals die bisherige Ansicht über die Quelle der Vie Sainte Elysabel nicht mehr haltbar ist.

Rutebeuf selbst spricht von seiner Quelle in den Versen

28. (Krefsner) de li (Elysabel) list on en sainte yglise.

2142.

tote trere

de latin en rime francoise.

22. me vueil je entremetre

de ceste estoire en rime metre.

Diese Angaben zeigen also, dals Rutebeuf einen lateinischen Prosatext versifiziert hat, der bald nach Elisabeths Tode veröffentlicht sein muls, sodafs Rutebeuf ihn in der zweiten Hälfte des 13. Jahrhunderts bereits benutzen konnte.

Elisabeth, eine böhmische Prinzessin, wurde 1207 geboren und kam im Alter von vier Jahren an den Hof des Landgrafen von Thüringen, mit dessen Sohn Ludwig sie nach damaliger Sitte bald nach ihrer Geburt schon verlobt worden war. Die beiden Fürstenkinder heirateten sich im Jahre 1220. Einige Jahre später erhielt Elisabeth Konrad von Marburg als Beichtvater, dessen Einflufs seine Höhe erreichte, als Ludwig 1225 wegen der politischen Ereignisse jener Zeit ein Jahr in Oberitalien weilen mufste. Während seiner Abwesenheit zeigte Flisabeth ihre Mildthätigkeit und Güte bei einer Hungersnot, die in Thüringen ausgebrochen war. Ludwig kehrte im Sommer 1226 in seill Iand zurück, aber schon 1227 nahm er von seiner Frau Abschied, um sich in Unteritalien als Kreuzfahrer einzuschiffen. Bevor er diese Absicht ausführen konnte, raffte ihn ein starkes Fieber hinweg. Sein Leichnam wurde der Wittwe überbracht, die gelobte, sich nicht wieder zu verheiraten. Sie entsagte der Regierung des Landes und ihren Besitztümern zu Gunsten ihrer Kinder und siedelte nach Marburg über, wo sie in den Franziskanerorden eintrat. Auch hicr zeigte sie sich als eine wahre Mutter der Kranken und Armen, bis sie im Jahre I 23 I starb. Meister Konrad überlebte sie nur kurze Zeit, indem er 1233 von einigen Rittern ermordet wurde, die er der Ketzerei beschuldigt hatte. Während der beiden Jahre nach Elisabeths Tode hatte sich Konrad eifrig damit beschäftigt, ihre Heiligsprechung durch Papst Gregor IX. zu erwirken. Mit Unterstützung des Erzbischofs Sigfried 
von Mainz sammelte er alles, was er über Leben und Wunder Elisabeths in Erfahrung bringen konnte, und richtete dann an den Papst ein Schreiben, worin er 37 wohl bezeugte Wunder Elisabeths aufführte, nachdem er einen kurzen Lebensabrils seines Beichtkindes vorausgeschickt hatte. Nur der Tod Konrads verhinderte, dafs diese „Epistola Magistri Conradi de Marburch ad Papam de vita Beatae Elisabethae" in die Hände Gregors kam. Aber Meister Konrad blieb nicht ohne Nachfolger in seinen Bemühungen. Der Herzog Konrad, Schwager Elisabeths und Vormund ihrer Kinder, der kurz zuvor Mitglied des Deutschordens geworden war, richtete an den Papst die Bitte, er möge Elisabeth kanonisieren lassen. Gregor beauftragte eine Kommission von drei Geistlichen, von neuem die der Elisabeth zugeschriebenen Wunder zu prüfen. Dies geschah, und Konrad selbst überbrachte das Resultat der Nachforschungen dem Papste, der Elisabeth Pfingsten I 235 heilig sprach.

Die beiden Dokumente, die dem Papste die Würdigkeit Flisabeths, heilig gesprochen $\mathrm{zu}$ werden, beweisen sollten, sind uns erhalten. Das eine, der Brief Meister Konrads 1, hat seine ursprüngliche Form nicht geändert, während das zweite, der Bericht Herzog Konrads, benutzt ist in dem „Libellus de Dictis quattuor ancillarum Sanctae Elisabethae sive Examen miraculorum eius". 2 Dieser Libellus wurde bisher als Quelle von Rutebeufs Gedicht angesehen. Man verliefs sich dabei auf P. Paris, der dies Quellenverhältnis in der Histoire littéraire XX, p. 780 durch seine Autorität sanctioniert hatte. In der That zeigen der Libellus und Rutebeufs Gedicht, das ich fortan kurz als ,Vie' bezeichnen will, eine frappante Aehnlichkeit, sodafs man leicht $\mathrm{zu}$ dem Glauben verleitet wird, dafs Rutebeuf den Libellus wirklich benutzt hat. Ich citiere zunächst einige Stellen, deren Uebereinstimmung dem Zufall jedenfalls nicht zugeschrieben werden kann.

Libellus.

p. 2007 A. (Gregorius), quem patrem urbi et orbi ${ }^{8}$ divina providit ordinatio.

2011 A. actum Perusii anno gratiae 1234.

2012 A. (miracula) per testes idoneos sollemniter et suffcienter probata, quae in Curia Romana examinata sunt et insuper diligentissime approbata.
Vie.

v. 47. droiz peres en verité et au pueple et a la cité.

121. ce fu doné a la Parrousse en l'an de l'incarnacion mil et deus cent et quatre et trente

si com l'escripture le chante.

23I. miracles....

que cil de sa voisinité que furent creable et preudome proverent a la cort de Rome.

1 Hrsg. von Kuchenbecker, Analecta Hassiaca III, p. 107-I47.

Hrsg. von Mencken, Scriptores Rerum Germanicarum II, p. 2007-2034.

8 Die übereinstimmenden Worte sind cursiv gedruckt. 
Libellus.

p. 2012 B. haec requisita. jurata dixit.

2012 C. ore pavimento adhaerens. 2012 D. de lucro suo dabat decimam pauperibus puellulis obligans ad dicendum aliquot Pater Noster cum Ave Maria.

2014 A. Isentrudis vidua religiosa de Hursilgove, quae fuit in familia beatae Elisabeth circiter quinque annos vivente marito eius Ludovico landgravio. humilem et valde caritati. vam.

2015B. de suis grangiis specialibus.

2019 A. in his omnibus praedicta Guda et Ysenstrudis juratae concordant.

2022. duo milia marcarum.

2026 B. duodecim denarios Coloniensis monetae.
Vie.

v. 267. a cele vierge fu requis.

273. cele jura et dist apres.

291. au pavement joignoit sa boche. 317. aus povres puceles meisme de trestot son gaaing la disme. 320. dire devoit la patre nostre et le salu la Dame qui tant a valu. 491. Ysentruz qui fu veve fame relegieuse et bone dame, fu avoec li cinq ans, ce croi, de son conseil, de son secroi au vivant Loys landegrave.

506. humble, pleine de charité.

848. de ses granges especiaus.

1003. ces deus dames qui juré orent qui la vie a la dame sorent s'accorderent si bien ensamble.. 1283. deus mille mars.

1545. tot douze colognois.

Wie steht es nun mit der Disposition in beiden Texten? Folgende Tabelle wird darüber Auskunft geben.

\section{Libellus.}

p. 2007-201 A : Eriahlungr der Kianonisation.

2011 A -2011B : Entschuldigung des Verfassers wegen der Komposition seiner Erzäblung.

2011 C-2012A: Entschuldigung des Verfassers wegen seines Stils.

2012 A :
Vie.

v. I -40 : Einleitung: Veranlassung des Gedichts.

41-126: Erzählung der Kanonisation.

127-208: Prolog zu den Zeugenaussagen.

\footnotetext{
209-234: Inhaltsübersicht.

235-254: Uebergang zum ersten Teile.
} 
Libellus.

p.2012B-2014A : Erster Teil:

Leben Elisabeths bis

zu ihrer Heirat.

2014B-2019A: Zweiter Teil:

Elisabeths Ehe.

2019B-2024A: Dritter Teil:

Wittwenschaft Elisa-

beths bis zum Ein-

tritt in den Fran-

ziskanerorden.

2024A-2032 B: Vierter Teil:

Die letzten Jahre

Elisabeths.

2032C-2034: Einige Wunder Eli-

sabeths.
Vie.

v. 255-436: Erster Teil.

437-480: Uebergang zum zweiten Teile.

481-999: Zweiter Teil.

1000-1032: Uebergang zum dritten Teile.

1033-1392: Dritter Teil.

1393-1402: Uebergang zum vierten Teile.

1403-2016: Vierter Teil.

2017-2119: Einige Wunder Elisabeths.

2120-2162: Persönliche Bemerkungen des Dichters.

Wenn wir absehen von den Stellen, welche Rutebeuf selbst eingefügt hat (Einleitung, Uebergänge, Schlufs), so erkennen wir, dafs die Disposition beider Texte dieselbe ist. Nur die Seiten 201 I bis $2012 \mathrm{~A}$ des Libellus stimmen nicht mit den Versen $127-208$ der Vie. Hierin liegt nun das punctum saliens. Im Libellus heifst es in der Mitte der beiden Entschuldigungen, die der Verfasser dem Leser gegenüber für nötig hält (2011 B):

,In prologo continentur inanes laudum tituli et taediosae virtutum Elisabethae repetitiones, quas pace lectoris omittimus".

Wir erfahren also, dafs der Verfasser des Libellus in seiner Quelle einen Prolog fand, der den Zeugenaussagen der vier Dienerinnen voranging. Da wir nun gesehen haben, dals die beiden Texte sowohl in der Disposition als auch in vielen Ausdrücken gleich sind, so scheint es mir sicher zu sein, dals der Prolog der Vie (v. 127 bis 208) uns die Uebersetzung der Stelle giebt, die der Verfasser des Libellus zurückgewiesen hat. Wirklich finden sich in der Vie viele Ausdrücke, die in anderen Teilen derselben wiederkehren, so z. B. in den Versen

167. desqu'ele n'avoit que cinq anz (Prolog).

260. des les cinq anz et puis en ca (Erster Teil). 
147. au roi de Hongrie fu fille (Prolog).

258. la fille le roi de Hongrie (Erster Teil).)

157. toz vices de sa vic osta (Prolog).

173. n'osta pas bien vices de li

cele....

? (Prolog)

189. osta si et mist a senestre (Prolog).

Interessantes lehren uns ferner die Verse I9I-193:

por ce que sermoner me grieve,

le prologue briefment achieve,

que ma matire ne destruie.

Wir sehen also, dafs selbst der naive französische Dichter die Schwächen des von ihm benutzten Prologs erkennt. Aber er hat nicht den Mut gehabt, diese Stelle aus der Vie herauszulassen, wie es der Verfasser des Libellus seinerseits gethan hat. Letzterer hat seine Kritik noch weiter ausgedehnt. Er ist mit der Komposition und der Diction seiner Quelle, deren Prolog er bereits verschmäht hat, nicht zufrieden und glaubt dem Leser gegenüber einer Entschuldigung dafür zu bedürfen. Jedenfalls ist er ein gebildeter Mönch (20 I 2A Tulliana eloquentia), dessen Stilgefühl die den Legenden eigene Einfachheit des Inhalts und des Ausdrucks nicht liebt. - Aus dem bisher gesagten ergiebt sich, dafs der Libellus nicht die Quelle der Vie sein kann, weil ihm der in der Vie erhaltene Prolog fehlt, dafs aber andererseits Libellus und Vie auf eine gemeinsame Quelle zurückgehen. Die ursprüngliche Komposition findet sich in der Vie, aber die Aehnlichkeit vieler Ausdrücke im Libellus und in der Vie beweist, dals, ausgenommen Prolog und Entschuldigungen, der Libellus im allgemeinen die Gestalt und den Text der lateinischen Quelle darbietet, die Rutebeuf zu seiner Verfügung hatte.

Wir können daher mit ziemlicher Sicherheit sagen, wie Rutebcuf seine Quelle benutzt hat. Im allgemeinen versucht er seiner Erzählung den Charakter einc's historischen Dokuments zu nehmen und bedient sich der naiven und volkstümlichen Sprache der Legenden. Finige Verïnderung(m), die sich in der Vic finden, leiten sich aus dieser Tendenz her. Zunächst ändert er Eigennamen: aufser Elysabel findet sich auch Ysabiaus (v. 37 ; 200; 1437; 1 555); Guda (2024 A) wird Gronde (1309); Hildegundis (2025 B) Herluiz (1453; I 500); Panian (2028 I3) Pavien (1688); Gerardus (2029 B) Gautier (175.5); auch im ersten Teil von Elisabeths Leben (2012 bis $2014=$ V. 255 bis 436 ) ist der Name der Zeugin verschieden: der Verfasser des Libellus nennt sie Guda, der Dichter der Vie: Elisabeth. - Einige Male übersetzt Rutebeuf den Text seiner Quelle durch volkstümliche Wendungen:

$$
\text { Libellus. }
$$

p. 2015 B. equitabant eodem die octo miliaria teutonica, quae large faciunt triginta italica.

\section{Vic.}

v. 652. puis les covint il errer tels huit lieues que par droit conte l'une de la, deus de ci monte. 
Libellus.

p. 2026 B. duodecim denarios Coloniensis monetae.

2033 B. haec famula tredecimo calendas decembris decessit.
Vie.

v. 1545. avoec tot douze coloignois dont li uns vaut quatre tornois.

2070. trespassa tot droit l'endemain

des octaves la Saint Martin.

Oft schiebt der Dichter in die ihm vorliegende Quelle Wortspiele moralisierenden Inhalts ein, daneben auch persönliche Bemerkungen bezüglich seiner Quelle, um seine Erzählung mehr authentisch zu machen. Wir kennen den Wert solcher Versicherungen bei altfranzösischen Dichtern zur Genüge, und auch in der Vie sind diese Citate oft nichts als Phrasen, so in den Versen 26, 40, 148, 154, 1032, 1409, 1586, 1685, 2148 . Uebrigens finden sich auch eine Menge richtiger Citate, die man im Libellus wiedererkennt, so V. $126=$ p. $2011 \mathrm{~A} ; 210=2012 \mathrm{~A} ; 255=2012 \mathrm{~B} ; 547=2014 \mathrm{~B}$; $946=2018 \mathrm{C} ; 1309=2022 \mathrm{~B} ; 1662=2028 \mathrm{~A} ; 2078=2033 \mathrm{C}$. Der Bibelvers (2. Thess. 3, 10), der sich in Vers 2 der Vie findet, ist auch wohl von Rutebeuf seiner Quelle entlehnt, da er auch im Libellus steht (2018 B).

Die übrigen Aenderungen sind nicht von grofser Wichtigkeit. Einige Stellen sind etwas abgekürzt, so $2013-2013 \mathrm{~B}=337-35^{6}$ (der hl. Johannes wird Schutzpatron der Elisabeth); 2015 A= 623-626 (wie Elisabeth ihren Dienerinnen Brot verschafft). Im ersten Teil der Erzählung (V. 255-436) hat Rutebeuf die Reihenfolge zweier Erzählungen geändert (p. 201 $2 \mathrm{C}=\mathrm{V}$. 323-336; p. $2012 \mathrm{D}=\mathrm{V}$. 315-322); es scheint, dals der Dichter durch ihre gleichen Anfänge dazu veranlalst ist; die eine Erzählung beginnt $(2012 \mathrm{C})$ : "item in ludo annulorum et quolibet alio ludo", die andere : „item in ludo annulorum et de quolibet alio lucro". - Das Verzeichnis der zu Ehren Elisabeths gelesenen Messen ist ausgelassen, da Rutebeufs Erzählung einen anderen Zweck als die einfache Legende verfolgt. Mehrere Male hat auch der Dichter seine Quelle falsch übersetzt. So heifst es im Libellus (2015 B):

„item tempore generalis famis et caristiae, landgravio profecto ad curiam Cremonensem, omnem annonam (Elisabeth) de suis grangiis specialibus collectam in pauperum elemosinis expendit“.

\footnotetext{
Rutebeuf übersetzt (V. 843-85I):

Or fu jadis en un termine Que il estoit molt grant famine; Landegrave, qui preudom iere Et qui l'amor Dieu avoit chiere, Envoia com preudom loiaus

De ses granges especiaus

Tot le gaaignage a Stremone, Sanz ce que uns ne l'en sermone, Por departir aus povres genz.
} 
Eberiso falsch übersetzt ist dic Stelle (2017 C):

„sub castro Wartberch altissimo erat magna domus, in qua plurimos reponebat infirmos, qui generalem elemosynam non poterant exspectare, quos singulis diebus visitabat".

Bei Rutebeuf heifst es (V. 855-865):

A Watebort demoroit lors

Un chastel de la vile tors;

Leenz a une grant meson,

Qui lors estoit en la seson

Plaine d'enfermes et d'enfers;

Assez estoit griez cis enfers.

Cil ne pooit pas tant atendre

Cele ore a quoi l'en soloit rendre

Aus povres l'aumosne comune,

Mes ja n'i eust un ne une

Qu'il ne veist chascun par soi.

Diese Angaben werden genügen, um die Beziehungen der Vie zu ihrer Quelle zu charakterisieren; wir wissen bereits, dals letztere auch dem Verfasser des Libellus vorgelegen hat, und wollen versuchen, dieselbe, soweit es möglich, wieder $\mathrm{zu}$ reconstruieren.

Man möge sich des bereits erwähnten Berichts erinnern, der dem Papst durch die Kommission der drei Geistlichen gesandt wurde. Es ist klar, dafs die Zeugenaussagen der Dienerinnen (2012 B-2032A) mit diesem Bericht identisch sind. Er bildet also die Grundlage des Libellus, aber er war ursprünglich länger: hinter den Zeugenaussagen fanden sich etwa 150 Wunder der Elisabeth ${ }^{1}$ aufgezählt, dazu die Namen aller derer, die die Kommission unterstützt hatten, die Wahrheit der Wunder zu prüfen. Da weder der Libellus noch die Vie Namen und Wunder enthalten, so können wir annehmen, dafs die gemeinsame Quelle beider Texte sie auch nicht hatte. Rutebeuf behauptet allerdings (V. 77-84), in seiner Quelle die Namen jener Geistlichen selbst gelesen zu haben, aber diese Versicherung scheint mir ziemlich unwesentlich zu sein. Von diesem Berichte gilt is nun eine Brücke zu finden hinüber zu dem Libellus und der Vie.

Bald nach der Heiligsprechung Elisabeths benachrichtigte der Papst alle Gläubigen davon durch ein Breve, welches uns erhalten ist. ${ }^{2}$ Dieses Dokument wird auch im Libellus erwähnt (20 I I A): „Multa vero litterarum paria beatissimus Papa non tantum per sacrum Imperium, verum et per omnia mundi climata, regna et metropoles dirigebat, gestorum sollemnia praetaxatorum publicans et edicens, quae diligenter inspecta ipsius circa praesens nego-

$12012 \mathrm{~A}$ (Inhaltsverzeichnis): ultimo annexa sunt miracula, quae post mortem Dominus ad tumbam eius pro ipsa operatus est manifeste. Et sunt ex infinitis quaedam electa circiter CL per testes idoneos sollemniter et sufficienter probata.

2 Uebersetzt bei Montalembert, Histoire de Sainte Elisabeth p. 602-6ro. 
tium fervidam sinceritatem et sincerum favorem copiosius ac plenius declarabunt. Actum Perusii anno gratiae MCCXXXIV“. Dieses Datum, das sich auch am Schlufs des Breve findet, zeigt, dafs der Verfasser dieser Stelle jenes Dokument vor sich hatte. Aber abgesehen von diesem offiziellen Breve beeilte man sich natürlich, die Wunder der neuen Heiligen in legendarischer Form den Gläubigen mitzuteilen. Man kann nicht zweifeln, dals die Quelle des Libellus und der Vie diese Legende ist. Vielleicht war der Verfasser ein Geistlicher aus der päpstlichen Kanzlei, jedenfalls aber ein Mann, der die offiziellen Schriftstücke, das Breve und den Bericht der drei Geistlichen, zu seiner Verfügung hatte. Eine Stelle des Libellus scheint darauf hinzuweisen, dafs er Italiener war. ${ }^{1}$ Auch wird die Autorität des heiligen Stuhles mehrfach hervorgehoben. Der Verfasser dieser Legende fügte dem Bericht der drei Geistlichen hinzu: einen kurzen Abrifs der Kanonisation, den Prolog, die Inhaltsübersicht und ein Verzeichnis der vom Papst befohlenen Messen. ${ }^{2}$ Von 150 Wundern suchte er die hauptsächlichsten aus ${ }^{3}$ und strich die Namen der Geistlichen, die die Wahrheit der Wunder geprüft hatten. Dieser legendarische Bericht wurde einige Zeit nach der Kanonisation in alle Länder vertrieben, sodafs er auch nach Deutschland und Frankreich gelangte. - Das Affiliationsverhältnis der besprochenen Schriften ist mithin folgendes:

Offizieller Bericht der drei Geistlichen.

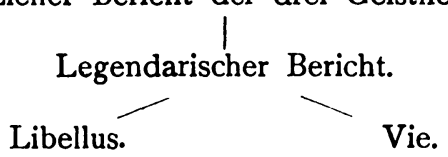

1 p. 2015 B: equitabant eodem die octo miliaria teutonica, quae large faciunt triginta italica.

2 p. 2010B: officium missarum, quod in fine huius legendae abbreviatum reperitur.

8 Der Verfasser beabsichtigt eine vollständige Ausgabe der Wunder (p. $2033 \mathrm{~A}$ cuius rei processum et seriem alias credimus persequendum).

Carl Frieslani). 